\title{
Quatre questions pour rallier les parties prenantes à la gestion d'un projet ${ }^{1}$
}

\author{
Lavagnon Ika ${ }^{a}$, Jan Saint-Macary ${ }^{b}$, Alassane Bandéc
}

RÉSUMÉ. Les parties prenantes constituent un élément clé pour la réussite ou l'échec d'un projet. Pour en assurer une gestion plus efficace, cet article propose une approche qui tient compte des aspects politiques du projet. S'appuyant sur un cadre théorique et deux cas réels, il pose quatre questions clés pour mieux identifier, évaluer, mobiliser et gérer les parties prenantes, sur une base continue. Cette approche instrumentale se concentre sur les profils des parties prenantes et sur la distinction entre leurs positions déclarées et leurs intérêts réels. Ainsi, elle permet de mieux gérer les parties prenantes existantes, d'en inclure de nouvelles au besoin, et de modifier le contexte politique du projet. En ralliant les parties prenantes et en se concentrant sur la satisfaction de leurs intérêts réels - mais souvent voilés - le projet peut être coproduit, ce qui se traduit par une situation « gagnant-gagnant » pour tous.

ABSTRACT. Stakeholders are undeniably a key component in the success or demise of projects. This article proposes an instrumental approach for a more effective management of stakeholders, by taking into account the rational as well as the political aspects of projects. Using an explicit theoretical framework and illustrations from two real-life cases, it builds on four key questions to identify, assess, engage, and manage stakeholders on an ongoing basis. This novel approach focuses on the profiles of the stakeholders and on the distinction between their stated positions and their real interests. It allows for the management of existing stakeholders and the inclusion of new ones, as required for the success of the project. By partnering with stakeholders and focusing on satisfying their real - but often veiled interests, the project can be co-produced, resulting in a "win-win" for all.

\section{Obama se fait dire «No, you can't»}

«Barack Obama n'est plus prophète à Chicago » (Lesnes, 2018). Cela n'est pas le titre d'un film, mais celui d'un article de journal. On y fait état du projet de construction de la bibliothèque présidentielle de Barack Obama à Chicago, qui, étonnamment, est chaudement contesté par les résidents. Inquiets des risques d'embourgeoisement de leur quartier, plusieurs d'entre eux se sont en effet mobilisés.

Pourtant, ce projet n'avait au départ rien d'inusité. En effet, il est de coutume que les présidents américains construisent une bibliothèque pour abriter leurs archives présidentielles quand ils quittent la Maison-Blanche. Obama, qui ne déroge pas à la règle, s'est associé à l'Université de Chicago pourlancer son projet dans le South Side de
Chicago plutôt qu'à Honolulu, son lieu de naissance. Quoi de plus normal que d'installer sa bibliothèque dans cette partie sud de Chicago habitée à $93 \%$ par des Afro-Américains, où il a été travailleur social, a fait ses débuts en politique et où on a massivement voté pour lui en 2008 et 2012? Tout était donc réuni pour que le projet passe comme « une lettre à la poste » (Lesnes, 2018).

Contre toute attente, ce projet se heurte à une forte opposition dans la communauté afro-américaine, pourtant partisane de l'ex-président. Il mobilise contre lui des «parties prenantes », au sens large de Freeman (1984), dont des associations de quartier et des syndicats de locataires. Des professeurs et employés de l'Université de Chicago ont même qualifié le projet de « régression sociale ». D’autres opposants sont persuadés que le complexe présidentiel ne va

\footnotetext{
a Professeur de gestion de projet., École de gestion Telfer, Université d'Ottawa

b Professeur de stratégie, Département des sciences administratives, Université du Québec en Outaouais

${ }^{\mathrm{C}}$ Doctorant en gestion de projet, Université du Québec à Chicoutimi
} 
qu'accélérer une dépossession programmée par la mairie et l'Université. Pire, certains vont jusqu'à l'accuser de «nettoyage ethnique ». À ces récriminations s'ajoutent les griefs concernant le coût exorbitant du projet et les terrains nécessaires à sa réalisation, gracieusement offerts par la mairie de Chicago, dont le maire, Rahm Emanuel, a été le premier chef de cabinet de l'ex-président Obama.

Manifestement, le gestionnaire de projet n'a pas effectué l'identification nécessaire de ces parties prenantes ni une analyse de leurs attentes et intérêts. Sur la base du soutien apporté au candidat Obama, les concepteurs du projet croyaient en connaitre les parties prenantes et anticipaient même leur appui. Or, leurs attentes n'ont pas été satisfaites lors du passage de l'ex-président à la Maison-Blanche. Visiblement, ce projet présentait, pour cette communauté, des enjeux différents de la double élection d'Obama : "Yes you can, $M r$. President» à l'échelle nationale, "No, you can't» à l'échelle municipale.

Les concepteurs ont donc erré en tenant pour acquis l'appui de cette communauté pour tout ce qu'Obama entreprendrait. Pourtant, certains indices portaient à penser que leur soutien n'était pas inconditionnel. «Nous avons eu assez confiance en lui pour l'élire à la Maison-Blanche, non pas une fois, mais deux, et nous ne lui ferions pas confiance, ni à Michelle, pour faire le bien dans leur propre communauté » (Lesnes, 2018, s. p.), exprimait un membre de cette communauté. Le révérend Finley Campbell n'at-il pas affirmé que la présidence de Barack Obama a apporté un changement positif, voire un triomphe pour les élites et la bourgeoisie noires, mais qu'elle a laissé tomber la classe ouvrière?

Ce projet, qui illustre plusieurs problèmes de ralliement des parties prenantes soulevés dans la littérature universitaire et professionnelle, n'est pas un cas isolé (Cleland, 1986; Littau, Jujagiri et Adlbrecht, 2010; PMI, 2017; Winch, 2017). Il serait donc souhaitable de convertir l'intérêt et l'influence des parties prenantes en participation active, et d'obtenir ainsi de meilleurs résultats pour tous. En effet, l'adoption d'une approche proactive, plutôt que réactive, de gestion avec les parties prenantes serait pertinente pour combler les attentes des parties prenantes (Eskerod et Huemann, 2014). Dans cette perspective, les praticiens doivent absolument se poser un certain nombre de questions pour rallier les parties prenantes au projet.
Cet article s'appuie sur quatre questions de départ, formulées de manière à les inscrire dans une démarche concrète de mise en œuvre d'un projet (Frooman, 1999; Carroll et Näsi, 1997; Pinto, Slevin et English, 2009) :

1) Qui sont les parties prenantes et que veulent-elles? 2) Quels pouvoirs et quelle influence détiennent-elles, et comment les exercent-elles? 3) Comment modifier le contexte politique du projet? 4) Comment gagner et gérer l'adhésion des parties prenantes?

En premier lieu, nous présentons succinctement nos repères théoriques, puis nous abordons tour à tour les quatre questions essentielles que nous suggérons pour rallier les parties prenantes.

\section{Les repères théoriques retenus}

La théorie des parties prenantes remonte au début des années 1960, notamment à un mémorandum interne du Stanford Research Institute. C'est à Freeman (1984) que revient le mérite d'avoir popularisé cette théorie et d'en avoir fait une des bases de la gestion stratégique moderne. Compte tenu du rôle prépondérant que jouent les projets dans la mise en œuvre de la stratégie de l'organisation, la théorie des parties prenantes a été vite reprise en gestion de projet, notamment avec les travaux précurseurs de Cleland (1986).

L'expression «partie prenante » suscite souvent une certaine confusion (Littau et collab., 2010). Comme Freeman (1984), le Project Management Institute (PMI) considère les parties prenantes comme «les personnes, les groupes ou organisations susceptibles d'affecter ou d'être affectés par le projet » (PMI, 2017, p. 503). En général, l'analyse des parties prenantes comprend quatre étapes principales : 1) l'identification, 2) l'évaluation, 3) l'ordonnancement et 4) la planification de la mobilisation ou de la démobilisation des parties prenantes (Eskerod et Huemann, 2014). Cette démarche peut être élargie en y incluant l'apport de nouvelles parties prenantes qui pourrait être suscité au besoin.

Par ailleurs, le ralliement des parties prenantes peut être envisagé comme un processus par lequel, de manière itérative, les parties prenantes sont cartographiées, de nouvelles parties prenantes sont sollicitées au besoin, et le pouvoir, la prise de position et l'intérêt de chacune d'entre elles sont évalués, pour les rassembler autour du projet et pour modifier ainsi 
son contexte politique afin de le réussir. De même, la confiance revêt une importance capitale dans le ralliement des parties prenantes d'un projet (Pinto et collab., 2009). En outre, la performance d'un projet signifie différentes choses pour différentes parties prenantes à différents moments. Par conséquent, les points de vue des parties prenantes d'un projet sur sa performance sont souvent subjectifs, perçus, dynamiques et idiosyncrasiques, voire divergents, conflictuels et contradictoires (Ika, 2014). Ainsi, tous les projets ont des supporteurs et des opposants. Cet antagonisme découle du fait que la mise en œuvre de n'importe quel projet requiert l'allocation de ressources matérielles, financières et gestionnelles importantes. Ces dernières, étant affectées à un projet particulier, ne peuvent être utilisées à d'autres fins. Il s'ensuit que tout projet, quel qu'il soit, est susceptible de bénéficier à certains individus ou à certains groupes, au détriment de certains autres.

L'approche proposée dans cet article s'inspire en grande partie du critère du « qui et quoi comptent vraiment » de Freeman (1984), en particulier des travaux de Frooman (1999). Ce dernier postule en effet que le processus de gestion des parties prenantes répond principalement à trois questions, à savoir: 1) Qui sont les parties prenantes? 2) Que veulent-elles? 3) Comment s'y prennent-elles pour obtenir ce qu'elles veulent? Nous les incorporons dans les questions proposées et nous en élargissons la portée pour inclure une gestion continue des parties prenantes durant toutes les phases du projet.

Voici en bref la démarche qui permettra de concevoir et de mettre en œuvre une gestion stratégique des parties prenantes :

- cartographier les parties prenantes;

- évaluer les parties prenantes;

- modifier le contexte politique; et

- gérer la relation avec les parties prenantes sur une base continue et « gagnant-gagnant».

\section{Question 1: Qui sont les partie prenantes et que veulent-elles?}

Même si l'on reconnait que le gestionnaire de projet et son équipe ne sont pas les seules parties prenantes du projet, il n'est pas facile d'en dresser une liste complète. De plus, les identifier n'est qu'un premier pas, car il faut aussi savoir : Qui est pour le projet? Qui est contre? Qui pèse dans la balance? Qui peut mettre les bâtons dans les roues? En bref, qui sont les parties prenantes et que veulent-elles?

Pour bien identifier les parties prenantes se trouvant à l'intérieur et à l'extérieur de l'organisation, il est possible de s'inspirer de deux approches formelles d'identification: l'une circulaire (voir le cercle des parties prenantes de Bourne et Walker, 2006), l'autre matricielle (voir la matrice importance/position d'Aaltonen, Kujala, Havela et Savage, 2015). Le processus d'identification des parties prenantes pourrait également utiliser des techniques informelles, en se basant par exemple sur l'expérience intuitive de l'équipe de projet (Yang et Shen, 2015). Dans les publications universitaires et professionnelles, ces parties sont considérées comme primaires ou secondaires, selon que, respectivement, elles sont directement ou indirectement touchées par le projet. On parle aussi de parties prenantes formelles ou informelles selon qu'elles entretiennent ou non des relations contractuelles avec le projet (Newcombe, 2003; Freeman, Harisson, Wicks et De Colle, 2010; Aaltonen et Kujala, 2010).

Pour mieux identifier les parties prenantes, il est utile d'utiliser une liste de contrôle en se rappelant que plusieurs d'entre elles sont issues des groupes internes et externes suivants (voir tableau 1). Pour élargir le champ de réflexion et recenser le plus grand nombre possible de parties prenantes, il est également utile de penser aux six dimensions des divers contextes de tout projet, connues sous l'acronyme PESTEL : Politique, Économique, Sociale, Technologique, Écologique et Légale.

\begin{tabular}{|l|l|}
\hline \multicolumn{1}{|c|}{ Internes } & \multicolumn{1}{c|}{ Externes } \\
\hline - Haute direction, directeurs fonctionnels & - Clients, distributeurs et utilisateurs finaux \\
- Membres de l'équipe de projet & - Fournisseurs, sources de fonds ou d'information \\
- Employés, départements, syndicats & $\begin{array}{l}\text { - Concurrents } \\
\text { - Commanditaires }\end{array}$ \\
& $\begin{array}{l}\text { - Politiques, groupes sociaux, gouvernements } \\
\text { Commanditaires }\end{array}$ \\
\hline
\end{tabular}

Tableau 1 - Les parties prenantes internes et externes 
Plus concrètement, dans cette première étape d'identification des parties prenantes, chaque membre de l'équipe de projet est invité à dresser sa propre liste, qui devra comprendre toutes les personnes et organisations dont l'intérêt dans le projet peut être positif ou négatif. Les membres de l'équipe pourront aussi suggérer les noms d'autres organisations et de personnes en mesure de contribuer utilement à la confection de cette liste. En combinant leurs suggestions individuelles, le gestionnaire de projet pourrait préparer la plus longue liste possible. Cela est important, car la faute la plus grave est l'omission. Souvent, une seule partie prenante très motivée et bien mobilisée peut contribuer à la réussite du projet ou lui causer de sérieux torts.

La première partie de la question 1 « qui sont les parties prenantes? " permettra de colliger une longue liste sans engendrer nécessairement de grands débats. Il n'en sera pas de même pour la deuxième partie «que veulent-elles? », qui porte sur les attentes et les intérêts des parties prenantes. La matrice importance/ position d'Aaltonen et ses collaborateurs (2015), qui repose sur le modèle à trois attributs (pouvoir, légitimité, urgence) proposé par Mitchell, Agle et Wood (1997), pourrait être utile en ce sens. Toutefois, au lieu d'établir de simples moyennes entre les placements faits par différentes personnes, il est préférable de chercher à comprendre les raisons qui justifient les divergences entre leurs jugements individuels. Cela suscitera des discussions édifiantes et permettra de mieux saisir le contexte politique du projet. De plus, il est important de dresser une image plus complète des parties prenantes en procédant à une analyse de leurs relations interpersonnelles et de leur réseau social. Enfin, il est nécessaire de veiller aussi à identifier et à évaluer les parties prenantes non pas de façon statique, mais dynamique, et ainsi à tenir compte des modifications de leurs attentes au fil du temps. En effet, les intérêts des parties prenantes et leur capacité d'influencer le projet varient au fur et à mesure que le projet avance.

\section{Question 2: Quels pouvoirs et quelle influence détiennent ces parties prenantes, et comment les exercent-elles (opposition, véto, appui ou autorisation)?}

En pratique, cette question a toute son importance, dans la mesure où elle va permettre à l'équipe de pro- jet de se représenter les stratégies d'influence des parties prenantes et de préparer ses stratégies de réponse en conséquence (Aaltonen et collab., 2015). Il faut alors visualiser l'influence relative de chaque partie prenante ou analyser le potentiel d'impact qu'elle peut avoir sur les décisions relatives au projet (Bourne et Walker, 2006). Comme on peut le constater avec l'exemple du projet de bibliothèque de Barack Obama, les populations de Chicago utilisent certaines stratégies pour s'opposer au projet. L'intervention des médias donne de la visibilité et une certaine légitimité à leurs réclamations, ce qui peut entraîner des modifications dans la mise en œuvre du projet.

En outre, il faut chercher à connaitre les stratégies d'influence des parties prenantes externes qui sont les plus susceptibles de s'opposer au projet. Enfin, il faut porter une attention particulière aux intervenants qui disposent de ressources ou des intrants dont le projet a besoin, et garder à l'esprit que, même si certaines parties prenantes ne sont pas capables à elles seules d'influencer considérablement le projet parce que ne disposant pas de ressources ou d'intrants, elles peuvent parfois s'allier à d'autres parties prenantes qui ont ce pouvoir. En d'autres termes, on doit reconnaître que certaines parties prenantes utilisent des stratégies directes et les autres, des stratégies indirectes (Frooman, 1999). Les évaluations du degré de pouvoir et d'influence des parties prenantes susciteront sans doute beaucoup de débats. Là encore, il faut éviter d'établir de simples moyennes entre les placements figurant au diagramme et qui seront proposés par différentes personnes. Comme on l'a vu dans les discussions autour de la question 1 , il est toujours important d'examiner avec soin les raisons qui justifient des jugements divers sur le pouvoir et l'influence de certaines parties prenantes. Cela suscitera des discussions édifiantes et permettra de mieux appréhender le contexte politique du projet.

À ce stade-ci, nous allons illustrer l'application des deux premières questions proposées et nous préparer aux deux dernières. À ces fins, nous utiliserons le projet de lancement de la Ford Mustang, qui, avec plus de 10 millions de voitures vendues en 2019, compte plus de clubs de propriétaires que toute autre voiture au monde. Mené il y a de cela plus d'un demi-siècle, ce projet a été géré avec brio par Lee Iacocca, en relation avec des parties prenantes très puissantes qui auraient réussi à faire échec à la majorité des gestionnaires (voir encadré 1). 
Lancée en 1964, la Ford Mustang a connu dès son lancement un succès commercial si spectaculaire et si soutenu que, 55 ans plus tard, la compagnie ne juge plus nécessaire d’indiquer sur la carrosserie les mots « Ford » et « Mustang » : le logo emblématique, un cheval mustang en plein galop, suffit à identifier le produit aux yeux du public.

Pourtant, ce projet audacieux d'offrir « une voiture sport à un prix abordable », mené par Lee Iacocca, exdirecteur du marketing promu directeur général, a fait face à des opposants aussi nombreux que redoutables. Le plus féroce d'entre eux n'était nul autre que le supérieur immédiat de ce dernier, Henry Ford II, PDG de l'entreprise et petit-fils de son célèbre fondateur. Pour mener son projet à bon port, Iacocca a cajolé, neutralisé et contourné plusieurs intervenants. Il a su fomenter des coalitions entre certains d'entre eux et a même réussi à provoquer l'émergence de nouvelles parties prenantes, tant à l'intérieur qu'à l'extérieur de l'organisation.

\section{Un contexte organisationnel tiraillé}

À ses débuts, la société automobile Ford était connue comme une entreprise « axée sur la production », comme l'indiquait la boutade de son fondateur Henry Ford I dans son autobiographie : « Le client peut avoir une voiture de n'importe quelle couleur, pourvu qu'elle soit noire » (Carlson, 2007, s.p.). Cette vision s'est traduite par la production en série d'une gamme limitée de modèles à bon prix et au moindre coût.

Au début des années 1960, la Ford Motor Company se remettait finalement du fiasco gigantesque qu'elle avait connu avec le lancement de la Edsel, moins de 10 ans plus tôt. Fruit de l'imagination d'Henry Ford $\mathrm{II}^{2}$, qui était alors président, chef de la direction et président du conseil d’administration, le mot Edsel a figuré par la suite dans le dictionnaire Webster pour désigner « un produit, un projet, etc. qui ne sont pas acceptés par le public malgré des attentes élevées et des efforts promotionnels coûteux » (Carlson, 2007, s.p.). Ce revers commercial avait coûté à la compagnie la moitié des 650 millions de dollars recueillis quand elle a été cotée en bourse en 1956.

Moins de 10 ans plus tard, deux perspectives s'imposaient pour les projets de lancement de nouveaux modèles chez la Ford Motor Company : une perspective de production, héritée du passé, et une vision marketing, qui émergeait mais à grand-peine, depuis le « grand fiasco » d'Edsel.

La perspective de production, plus prudente, misait sur l'offre de nouveaux modèles que Ford pouvait fabriquer en modifiant le moins possible sa chaîne de montage. En s'appuyant ainsi sur une offre stable et restreinte de produits combinée à un contrôle rigoureux des coûts de production, cette perspective garantissait la rentabilité à moindre risque, vision farouchement partagée par les spécialistes de la finance. Dans le pire des cas, cette vision entravait l'offre de nouveaux produits et privilégiait celle de modèles plus adaptés aux exigences de la production qu’à celles du client.

Pour sa part, la perspective de marketing, plus audacieuse, favorisait plutôt le développement de produits novateurs qui répondaient aux souhaits et aux «besoins » même inavoués des clients. Elle visait ainsi autant l'augmentation de parts de marché déjà desservies que la conquête de nouveaux segments de marché. Poussée à l'extrême, cette vision encourageait l'utilisation d'astuces de toutes sortes - comme le financement à terme des achats - pour promouvoir la vente, plutôt que la qualité intrinsèque et à long terme des produits offerts.

Ces deux perspectives avaient chacune ses supporteurs et elles ne faisaient pas bon ménage dans l'organisation. Les luttes intestines sur la production de la Mustang au début des années 1960 illustrent ces divergences de vues entre les spécialistes au sein de l’organisation.

Le concept de la Mustang était le produit de l’imagination d'un ingénieur de Ford nommé Don Frey. Il avait conçu l'idée novatrice de construire une voiture sport abordable et à deux places pour faire concurrence à la Corvette de GM. Alors que Frey était le brillant technicien, Lee Iacocca personnifiait le marketing. Selon lui, il fallait tout axer sur le client, à qui il souhaitait offrir des options emballantes. Or, Iacocca était aussi l'homme politique par excellence qui savait que, pour obtenir ce qu'on veut, il faut savoir comment utiliser le pouvoir et l'influence.

Le public cible de la Mustang était le marché des jeunes, alors en pleine expansion. Les analystes de Ford estimaient à 50000 voitures vendues annuellement de ce modèle, norme qui garantissait, aux yeux de leurs experts en finance, des marges de profit intéressantes et à peu de risque. Ainsi, en règle générale, à moins qu'un nouveau produit ne soit susceptible de dépasser ce seuil minimal, les experts en finance le jugeaient trop risqué et donc " problématique ». 
Selon ces experts, le véhicule conçu par Frey appartenait malheureusement à cette catégorie de produits problématiques. Iacocca a donc proposé l'idée d'ajouter des sièges baquets à l'arrière de la Mustang et a ainsi réussi à convaincre le département de marketing de porter ses estimations à 100000 voitures vendues. C'était mieux, mais toujours insuffisant pour surmonter l'opposition farouche du département des finances, car ce modèle exigerait des modifications importantes à la chaîne de montage. Il y avait donc une double incertitude, tant au sujet des prévisions de ventes que des coûts de fabrication.

À ce stade, le défi de gestion des parties prenantes auquel Iacocca faisait face était en grande partie politique. Pour faire avancer les choses, il s'est évertué à contrecarrer l'opposition d'Henry Ford II, détracteur acharné, en l'impliquant graduellement dans l'évolution du projet. Évitant les canaux officiels, Iacocca partageait ses idées en primeur avec Ford II lors de rencontres informelles pour lui donner le temps de les évaluer et d'en parler aux autres. Son objectif était de permettre à Ford II de participer à l'évolution du concept et de faire progressivement sienne l'idée de la Mustang.

Or, cela s'est avéré plus difficile que prévu, car le département des finances continuait à s'opposer farouchement au projet en raison de son effet " problématique » sur la rentabilité de l'entreprise. Iacocca a alors pris le risque majeur de parler de la Mustang aux gens du siège social de Ford et aux membres du conseil d'administration. En parallèle, il a fait « fuiter » l'information sur la Mustang à la presse automobile spécialisée. Impatients d'en savoir plus sur cette nouvelle sensation automobile et sa date de sortie, des journalistes et des concessionnaires ont alors commencé à poser des questions aux dirigeants de Ford. Ainsi, de nouvelles parties prenantes ont émergé graduellement : le public, les concessionnaires Ford, le département de la production, le syndicat, les membres du conseil d'administration et les actionnaires.

À la veille du lancement de la Mustang, la direction du marketing avait maintenu sa prévision de ventes à 100000 voitures. La Mustang est sortie en 1964. Les ventes des premiers mois s'étant avérées fructueuses, Iacocca a persuadé la direction de confier la production de la Mustang à une deuxième usine, puis d'en construire une troisième, portant la capacité de production à 400000 voitures.

Fort de son succès, Iacocca a accédé ultimement à la présidence de la Ford Motor Company en 1970, sans jamais réussir à rallier pleinement Henry Ford II. Ce dernier l'a congédié en 1978, alors que la compagnie affichait des profits records de 2 milliards de dollars. Iacocca s'est par la suite rendu célèbre en devenant PDG de Chrysler, qu'il sauvera de la faillite.

Encadré 1 - Le projet de la Ford Mustang de Lee Iacocca et le défi du ralliement des parties prenantes Source : adapté de Carlson, 2007

\begin{tabular}{|c|c|c|c|c|c|}
\hline (Élevé) & & & $\begin{array}{l}\text { Conseil } \\
\text { d'administration }\end{array}$ & & \\
\hline \multirow{3}{*}{$\begin{array}{r}\text { POUVOIR } \\
\text { (influence) }\end{array}$} & Henry Ford II & & & & \\
\hline & & Finances & & & \\
\hline & & & & & $\begin{array}{l}\text { Don Frey } \\
\text { Marketing }\end{array}$ \\
\hline (Bas) & & & & Manufacture & \\
\hline
\end{tabular}

Tableau 2 - Première cartographie de la relation pouvoir/intérêt des parties prenantes au début du projet, du point de vue de Lee Iacocca, le gestionnaire de projet 


\section{Questions 3 : Comment modifier le contexte politique du projet (mobiliser, neutraliser, coopter ou faire émerger des parties prenantes)?}

Cette question se justifie particulièrement quand les attentes et intérêts des parties prenantes sont divergents, voire conflictuels, alors que les ressources pour y faire face sont limitées. C'est le cas de la Ford Mustang. On peut distinguer deux niveaux de ralliement des parties prenantes, à savoir : 1) l'implication des parties prenantes, quand on les informe et les consulte, et 2) la participation réelle, comme niveau plus élevé de ralliement des parties prenantes et qui permet de réduire leur résistance au projet. Puisque le ralliement des parties prenantes consiste en un processus de consultation, de communication, de dialogue et d'échange, la négociation devient de facto un impératif, compte tenu des divergences entre, d'une part, le projet et ses différentes parties prenantes et, d'autre part, entre les parties prenantes elles-mêmes. Seule la négociation permettra d'arriver à un compromis satisfaisant pour toutes les parties. En effet, le projet fait probablement face à ce que Freeman (1984) appelle " le dilemme de la partie prenante »: la négociation ou le conflit.

La communication est l'outil qui aidera à mener à bien le processus de négociation. D’où la nécessité de (Assudani \& Kloppenborg, 2010) mettre en place une stratégie et un plan de communication appropriés afin de s'assurer que les parties prenantes reçoivent les messages adéquats dans le bon format et selon la bonne fréquence. Les supports de communication peuvent entre autres inclure des courriels, des conversations informelles, des réunions formelles et des rapports réguliers du projet (PMI, 2017).

Revenons au cas de la Ford Mustang afin d'illustrer la mise en application de la question 3. Iacocca s'est attelé judicieusement à modifier le contexte politique dans lequel son projet avait tant de peine à avancer. Il y est parvenu en neutralisant partiellement certaines parties prenantes et en en cooptant d'autres, identifiées dans le tableau 2.

En dépit de ses sentiments à l'égard du projet, Henry Ford II est devenu graduellement un allié objectif de Iacocca. Peut-être que Ford II se remettait mal de l'échec commercial du projet Edsel, qu'il avait parrainé moins de 10 ans plus tôt. Toujours est-il qu'Iacocca a réussi à contenir son opposition à la Mustang en l'associant à ce projet très prometteur. Par la suite, le volume retentissant des premières ventes a scellé cette alliance basée sur leurs intérêts mutuels. En toute logique, avec des ventes de 22000 voitures le jour même du lancement de la Mustang, ni Henry Ford II ni le département des finances ne pouvaient plus se distancer de Iacocca.

Sans prendre de répit, Iacocca s'est évertué à faire émerger de nouvelles parties prenantes. Les articles qu'il avait « soufflés » à la presse ont attiré l'attention des membres du conseil d'administration, des concessionnaires de Ford, du département de la production et, au bout du compte, du syndicat, qui ont commencé à exprimer un intérêt favorable à l'égard du projet de la Mustang. Une deuxième, puis une troisième usine ont été construites pour satisfaire à la demande. Graduellement, le département de la production et le syndicat sont devenus de nouvelles parties prenantes, favorables au projet.

Ainsi, plus on porte un intérêt, positif ou négatif, à l'égard d'un projet, plus on est susceptible d'intervenir, en faveur ou contre lui. Par ailleurs, le degré d'influence ou de pouvoir qu'on détient est proportionnel à l'impact qu'on peut avoir sur l'avancement et l'issue d'un projet. En bref, l'intérêt est relié à la probabilité d'intervention, alors que le pouvoir l'est à son impact. Les gestionnaires de projet avertis doivent donc être attentifs à ces deux dimensions et amener les parties prenantes de leur position dans la partie inférieure gauche de la matrice vers le coin supérieur droit (voir tableau 3). Leurs efforts, sur ces deux voies, doivent tous viser le coin supérieur droit. 


\begin{tabular}{c|l|l}
\cline { 2 - 3 } (Élevé) & $\begin{array}{l}\text { Maintenir ces pp satisfaites } \\
\text { Relier leurs intérêts aux aspects du projet qui } \\
\text { leur sont bénéfiques }\end{array}$ & $\begin{array}{l}\text { Gérer ces pp avec attention } \\
\text { Inclure ces pp dans le processus de prise } \\
\text { de décisions majeures } \\
\text { (influence) }\end{array}$ \\
$\begin{array}{c}\text { POImpact } \\
\text { (Bas) }\end{array}$ & $\begin{array}{l}\text { Surveiller ces pp } \\
\text { Les recenser régulièrement et écouter les } \\
\text { «nouvelles » informelles qui circulent }\end{array}$ & $\begin{array}{l}\text { Maintenir ces pp informées } \\
\text { Susciter des coalitions pour accoître leur } \\
\text { influence }\end{array}$ \\
\hline
\end{tabular}

(Bas) INTÉRÊT $=>$ Probabilité d'intervention dans le projet

(Élevé)

Tableau 3 - Stratégies de communication et de ralliement des parties prenantes fondées sur la relation pouvoir/intérêt

Source partielle, assortie de commentaires : PMI (2017)

À titre d'exemple (voir tableau 4), Iacocca a su provoquer l'intérêt accru du conseil d'administration en faveur du projet. En utilisant les communications personnelles et en s'adjoignant la presse, il a pu aussi circonscrire quelque peu le champ d'action d'Henry
Ford II. De plus, en intéressant les concessionnaires Ford à cette nouvelle voiture sport, Iacocca a fait des jeunes acheteurs potentiels de la Mustang une partie prenante émergente.

\begin{tabular}{|c|c|c|c|c|c|}
\hline \multirow[t]{2}{*}{ (Élevé) } & & & $\begin{array}{l}\text { Conseil d'adminis- } \\
\text { tration }\end{array}$ & $\rightarrow$ & \\
\hline & $\begin{array}{l}\text { Henry } \\
\text { Ford II }\end{array}$ & $\longrightarrow$ & & & \\
\hline \multirow{2}{*}{$\begin{array}{r}\text { Pouvoir } \\
=>\text { impact } \\
\text { sur } \\
\text { le projet }\end{array}$} & & Finances & & & \\
\hline & & $\begin{array}{l}\text { Concessionnaires } \\
\text { Ford (clients) }\end{array}$ & & $\begin{array}{l}\text { Employés } \\
\text { (syndicats) }\end{array}$ & $\begin{array}{l}\text { Don Frey } \\
\text { Marketing }\end{array}$ \\
\hline \multirow{2}{*}{ (Bas) } & & & Presse & Manufacture & \\
\hline & (Bas) & Intérêt $=>$ Pro & abilité d'interventi & dans le proje & (Élevé) \\
\hline
\end{tabular}

5. Question 4 : Comment gagner et gérer l'adhésion des parties prenantes, en mode continu et de façon gagnant-gagnant (inclure et écouter les parties prenantes; évaluer et ajuster leurs rapports de force)?

En négociant dans une optique gagnant-gagnant, il y a lieu de distinguer la position adoptée par la partie prenante de ses intérêts réels. C'est en se concentrant sur ces derniers et en collaborant qu'on peut trouver ensemble des options novatrices et génératrices de gains mutuels (Fisher, Ury et Patton, 2006). Par exemple, la position très personnelle d'Henry Ford II contre la Mustang est essentiellement contraire à ses intérêts d'actionnaire, de PDG et de président du conseil d'administration. C'est le succès commercial de la
Mustang, combiné au fait que Iacocca l'y avait associé aux yeux du public, qui a porté Henry Ford II à modérer son opposition à ce projet.

En pratiquant une approche gagnant-gagnant, on arrive, au fil du temps, à établir un climat de confiance qui solidifie la relation du projet avec les parties prenantes, qui facilite la communication et qui raccourcit la durée des négociations. Plus les parties prenantes font confiance, plus elles partagent leurs connaissances et plus elles entreprennent des actions favorables au projet. Il devient alors possible de prendre des décisions plus rapidement, de réduire les coûts de transaction liés au suivi et au contrôle, et d'éviter des retards et des dépassements de coûts Pinto et collab., 2009. En outre, il est possible de tirer avantage du fait que les projets et leurs parties prenantes sont, à des degrés différents, interdépendants. Le projet a besoin 
des contributions financières et non financières des parties prenantes, tandis que ces dernières ont besoin du projet pour satisfaire certains besoins et attentes d'une autre nature.

La confiance n'est pas un concept binaire ou immuable, absolu ou inexistant. La confiance, ce n'est pas tout ou rien. Elle est plutôt une question de degré. De plus, elle évolue au fil du temps. L'objectif de l'équipe de projet devrait être de construire graduellement une confiance mutuelle, au fur et à mesure de l'avancement du projet. Dans le projet de construction de la bibliothèque présidentielle, il y a eu une certaine rupture de confiance entre l'ex-président des États-Unis et sa base. Cela s'est confirmé par le fait qu'on lui demande des engagements écrits, un community benefits agreement (CBA) (Lesnes, 2018). Barack Obama, à son tour, réclame plutôt la confiance. Il ne veut pas signer le moindre $\mathrm{CBA}$, alléguant que sa fondation est une organisation à but non lucratif, et non un promoteur immobilier dont il faudrait se méfier. En réalité, la confiance ne se réclame pas : elle se mérite. Il est donc très difficile de la rétablir lorsqu'on la perd.

\section{Conclusion}

Cet article a soulevé quatre questions fondamentales qu'il convient de se poser lorsqu'on cherche à obtenir l'adhésion des parties prenantes à un projet. Ces quatre questions concernent l'identification, l'évaluation, le ralliement et la gestion continue des parties prenantes et de leurs intérêts. Elles sont complémentaires et ne sauraient être abordées en silo. Elles doivent aussi être posées à répétition, sur une base itérative. Et c'est en les abordant de façon interdépendante qu'il est possible d'amener un projet au niveau de maturité le plus élevé possible de la gestion des parties prenantes, comme le vise le modèle de Freeman (1984). Le tableau 5 présenté ci-dessous propose un aide-mémoire pour les quatre questions et quelques recommandations destinées à aider à mieux réussir le ralliement des parties prenantes.

\begin{tabular}{|c|c|c|}
\hline Questions & Sous-questions & Recommandations \\
\hline \multirow[t]{3}{*}{$\begin{array}{l}\text { 1. Qui sont-elles et } \\
\text { que veulent-elles? }\end{array}$} & $\begin{array}{l}\text { Quelles sont les approches } \\
\text { formelles d'identification et } \\
\text { d'évaluation des pp? }\end{array}$ & $\begin{array}{l}\text { Plusieurs outils tels que le cercle des pp et la matrice } \\
\text { importance/position sont utiles, si on les met à jour } \\
\text { périodiquement. On peut aussi compter sur l'expérience } \\
\text { des membres de l'équipe et des pp formelles identifiées. }\end{array}$ \\
\hline & $\begin{array}{l}\text { S'agit-il de pp formelles ou } \\
\text { informelles? }\end{array}$ & $\begin{array}{l}\text { La plupart des organisations ne ciblent que les pp for- } \\
\text { melles, plus faciles à identifier. S'intéresser davantage } \\
\text { aux pp informelles, car elles sont les plus susceptibles } \\
\text { de s'opposer au projet. }\end{array}$ \\
\hline & $\begin{array}{l}\text { Prend-on en compte les aspects } \\
\text { dynamiques des pp et leurs } \\
\text { interactions? }\end{array}$ & $\begin{array}{l}\text { Les pp évoluent au fur et à mesure que le projet } \\
\text { progresse. En prendre continuellement la mesure et } \\
\text { tenir compte de leurs interactions. L'analyse des } \\
\text { réseaux sociaux peut aider dans ce sens. }\end{array}$ \\
\hline \multirow{3}{*}{$\begin{array}{l}\text { 2. Quels pouvoirs } \\
\text { et quelle influence } \\
\text { détiennent-elles, et } \\
\text { comment les } \\
\text { exercent-elles? }\end{array}$} & \multirow[t]{2}{*}{$\begin{array}{l}\text { Les pp disposent-elles des } \\
\text { ressources dont le projet a besoin? }\end{array}$} & $\begin{array}{l}\text { Si oui : s'attendre à ce qu'elles exercent directement } \\
\text { leur influence sur le projet. }\end{array}$ \\
\hline & & $\begin{array}{l}\text { Si non : penser aux alliances qu'elles peuvent nouer } \\
\text { avec les détenteurs de ressources et aux conséquences } \\
\text { pour le projet. }\end{array}$ \\
\hline & $\begin{array}{l}\text { A-t-on des stratégies de réponse } \\
\text { face à l'influence de certaines pp? }\end{array}$ & $\begin{array}{l}\text { Les stratégies de réponse doivent être individualisées } \\
\text { en tenant compte de la source d'influence et du niveau } \\
\text { de l'intérêt de chaque pp. }\end{array}$ \\
\hline \multirow{2}{*}{$\begin{array}{l}\text { 3. Comment } \\
\text { modifier le contexte } \\
\text { politique du projet? }\end{array}$} & $\begin{array}{l}\text { Quelles pp souhaite-t-on impliquer } \\
\text { modérément? }\end{array}$ & $\begin{array}{l}\text { Adopter une perspective à long terme, leur donner } \\
\text { l'heure juste et les consulter. }\end{array}$ \\
\hline & $\begin{array}{l}\text { Quelles pp souhaite-t-on impliquer } \\
\text { pleinement? }\end{array}$ & $\begin{array}{l}\text { Les écouter, respecter leurs valeurs, adopter leur } \\
\text { langage et leur donner surtout l'occasion de participer } \\
\text { à la prise de décision. }\end{array}$ \\
\hline \multirow{2}{*}{$\begin{array}{l}\text { 4. Comment gagner } \\
\text { et gérer leur } \\
\text { adhésion? }\end{array}$} & A-t-on confiance dans les pp? & \multirow{2}{*}{$\begin{array}{l}\text { Communiquer régulièrement avec elles. Se concentrer } \\
\text { sur leurs intérêts, plutôt que sur les positions adoptées. } \\
\text { La confiance s'installera progressivement. }\end{array}$} \\
\hline & A-t-on la confiance des pp? & \\
\hline
\end{tabular}

Tableau 5 - Récapitulatif des quatre questioins sur les parties prenantes 
Aussi cruciales soient-elles, ces quatre questions ne suffisent pas toujours pour rallier les parties prenantes. L'approche utilisée étant à la fois descriptive et instrumentale (Donaldson et Preston, 1995), elle fait l'impasse sur la dimension éthique de la théorie des parties prenantes (Derry, 2012). Malheureusement, il arrive souvent qu'en gestion de projet, on cherche encore à manipuler ou à défaut influencer les parties prenantes dans le seul intérêt du projet. Alors, n'est-il pas temps que, dans une perspective gagnant-gagnant, on veille à cocréer et à coréaliser le projet avec les parties prenantes? Ne sont-elles pas de véritables partenaires du projet, et non des objets que le gestionnaire de projet et son équipe peuvent manipuler à souhait (Woermann et Engelbrecht, 2019)? Voilà une réflexion à laquelle tout gestionnaire devra s'atteler pour mener à bien ses projets.

\section{NOTES}

1 Cet article a paru dans sa version anglaise en août 2020 dans la revue PM World Journal : https://pmworldjournal.com/ article/mobilizing-stakeholders-for-project-success

2 NDLR : Edsel Ford serait en fait le nom du grand-père d'Henry Ford II.

\section{RÉFÉRENCES}

Aaltonen, K. et Kujala, J. (2010). A project lifecycle perspective on stakeholder influence strategies in global projects. Scandinavian Journal of Management, 26(4), 381-397. https://doi.org/10.1016/j.scaman.2010.09.001

Aaltonen, K., Kujala, J., Havela, L. et Savage, G. (2015). Stakeholder dynamics during the project Front-End: The case of nuclear waste repository projects. Project Management Journal, 46(6), 15-41. https://doi.org/10.1002/pmj.21549

Bourne, L. et Walker, D. H. T. (2006). Visualizing stakeholder influence: Two Australian examples. Project Management Journal, 37(1), 5-21. https://doi.org/10.1177/875697280603700102

Carlson, P. (2007, Mardi, 4 Septembre). The flop heard round the world. The W ashington Post.

Carroll, A.B. et Näsi, J. (1997). Understanding stakeholder thinking: Themes from a Finnish conference. Business Etbics: A European review, 6(1), 46-51. https://doi-org.sbiproxy.uqac.ca/10.1111/1467-8608.00047

Cleland, D. I. (1986). Project stakeholder management. Project Management Journal, 17(4), 36-44. https:/ /www.pmi.org/learning/ library/project-stakeholder-management-5216

Derry, R. (2012). Reclaiming marginalized stakeholders. Journal of Business Ethics, 111(2), 253-264. https://doi.org/10.1007/ s10551-012-1205-x

Donaldson, T. et Preston, L. (1995). The stakeholder theory of the corporation: Concepts, evidence. Academy of Management Review, 20(1), 65-91. https://www.jstor.org/stable/258887?seq=1\#metadata_info_tab_contents

Eskerod, P. et Huemann, M. (2014). Managing for stakeholders. Dans J. R. Turner (dir.), Gower handbook of project management (5th ed.) (pp. 217-232). Farnham, GB: Gower.

Fisher, R., Ury, W. et Patton, B. (2006). Comment réussir une négociation (3e éd. française). Paris, France : du Seuil.

Freeman, R. E. (1984). Strategic management: A stakeholder approach. Boston, MA : Pitman.

Freeman, R. E., Harisson, J. S., Wicks, A. C. et De Colle, S. (2010). Stakeholder theory : The state of the art. Cambridge, GB: Cambridge University Press.

Frooman, J. (1999). Stakeholder influence strategies. Academy of Management Review, 24(2), 191-205. https://doi.org/10.5465/amr.1999.1893928

Ika, L. A. (2014). Grands projets : trop grands pour réussir? Revue Organisations \& territoires, 23(3), 15-24. https://doi.org/10.1522/ revueot.v23i3.137

Lesnes, C. (2018, 3 avril). Barack Obama n'est plus prophète à Chicago. Le Monde. Repéré à https://www.lemonde.fr/ameriques/ article/2018/04/03/obama-n-est-plus-prophete-a-chicago_5279795_3222.html

Littau, P., Jujagiri, N. J. et Adlbrecht, G. (2010). 25 years of stakeholder theory in project management literature (1984-2009). Project Management Journal, 41(4), 17-29. https://doi.org/10.1002/pmj.20195 
Mitchell, R. K., Agle, B. R. et Wood, D. J. (1997). Toward a theory of stakeholder identification and salience: Defining the principle of who and what really counts. The Academy of Management Review, 22(4), 853-886. https://doi.org/10.5465/amr.1997.9711022105

Newcombe, R. (2003). From client to project stakeholders: A stakeholder mapping approach. Construction Management and Economics, 21(8), 841-848. https://doi.org/10.1080/0144619032000072137

Pinto, J. K., Slevin, D. P. et English, B. (2009). Trust in projects: An empirical assessment of owner/contractor relationships. International Journal of Project Management, 27(6), 638-648. https://doi.org/10.1016/j.ijproman.2008.09.010

Project Management Institute (PMI). (2017). A guide to the project management body of knowledge (PMBOK Guide) (6th ed.). Philadelphie, PA: PMI.

Woermann, M. et Engelbrecht, S. (2019). The Ubuntu challenge to business : From stakeholders to relationholders. Journal of Business Ethics, 157(1), 27-44. https://doi.org/10.1007/s10551-017-3680-6

Yang, R. J. et Shen, G. Q. P. (2015). Framework for stakeholder management in construction projects. Journal of Management in Engineering, 31(4), 1-14. https://doi.org/10.1061/(ASCE)ME.1943-5479.0000285 